\title{
Article \\ Three Representation Types for Systems of Forms and Linear Maps
}

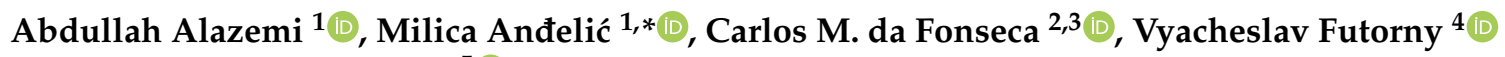 \\ and Vladimir V. Sergeichuk ${ }^{5}$ D \\ 1 Department of Mathematics, Kuwait University, Safat 13060, Kuwait; abdullah.alazemi@ku.edu.kw \\ 2 Department of Mathematics, Kuwait College of Science and Technology, Safat 13133, Kuwait; \\ c.dafonseca@kcst.edu.kw \\ 3 Chair of Computational Mathematics, University of Deusto, 48007 Bilbao, Spain \\ 4 Department of Mathematics, University of São Paulo, São Paulo 05508, Brazil; futorny@ime.usp.br \\ 5 Institute of Mathematics, Tereshchenkivska 3, 01024 Kiev, Ukraine; sergeich@imath.kiev.ua \\ * Correspondence: milica.andelic@ku.edu.kw
}

check for

updates

Citation: Alazemi, A.; Anđelić, M.;

da Fonseca, C.M.; Futorny, V.;

Sergeichuk, V.V. Three Representation

Types for Systems of Forms and

Linear Maps. Mathematics 2021, 9 ,

455. https://doi.org/10.3390/

math9050455

Academic Editor: Christos G

Massouros

Received: 6 January 2021

Accepted: 19 February 2021

Published: 24 February 2021

Publisher's Note: MDPI stays neutral with regard to jurisdictional claims in published maps and institutional affiliations.

Copyright: (c) 2021 by the authors. Licensee MDPI, Basel, Switzerland. This article is an open access article distributed under the terms and conditions of the Creative Commons Attribution (CC BY) license (https:/ / creativecommons.org/licenses/by/ $4.0 /)$.

\begin{abstract}
We consider systems of bilinear forms and linear maps as representations of a graph with undirected and directed edges. Its vertices represent vector spaces; its undirected and directed edges represent bilinear forms and linear maps, respectively. We prove that if the problem of classifying representations of a graph has not been solved, then it is equivalent to the problem of classifying representations of pairs of linear maps or pairs consisting of a bilinear form and a linear map. Thus, there are only two essentially different unsolved classification problems for systems of forms and linear maps.
\end{abstract}

Keywords: representations of quivers; representations of mixed graphs; wild problems

MSC: 15A04; 15A21; 16G20; 16G60

\section{Introduction}

We show that the problem of classifying pairs consisting of a bilinear form and a linear map plays the same role in the theory of systems of bilinear forms and linear maps as the problem of classifying pairs of linear maps plays in the theory of representations of finite dimensional algebras.

About fifty years ago, it was noticed that most unsolved classification problems in the theory of representations of groups and algebras "contain" the matrix pair problem, which is the problem of classifying pairs of square matrices of the same size up to similarity transformations

$$
(A, B) \mapsto\left(S^{-1} A S, S^{-1} B S\right), \quad S \text { is nonsingular. }
$$

(A classification problem $\mathcal{M}$ contains a classification problem $\mathcal{N}$ if solving $\mathcal{M}$ would solve $\mathcal{N}$.)

For example, Bašev [1] classified representations of the abelian group $(2,2)$ over a field of characteristic 2 . The problems of classifying representations of the abelian groups $(2,4)$ and $(2,2,2)$ over a field of characteristic 2 and the abelian group $(p, p)$ over a field of characteristic $p>2$ are considered as hopeless since these problems contain the matrix pair problem (Brenner [2] and Krugljak [3]).

Donovan and Freislich [4] call a matrix problem "wild" if it contains the matrix pair problem and "tame" otherwise, in analogy with the partition of animals into wild and tame ones. A certain characterization of tame and wild problems is given by Drozd [5] and Crawley-Boevey [6]; a geometric form of the tame-wild theorem is proved by Gabriel, Nazarova, Roiter, Sergeichuk, and Vossieck [7], and by Sergeichuk [8]. 
The reason for complexity of the matrix pair problem was found by Gelfand and Ponomarev [9], who showed that the problem of classifying pairs of commuting nilpotent matrices over any field up to similarity transformations (1) contains the problem of classifying matrix $t$-tuples up to similarity transformations

$$
\left(M_{1}, \ldots, M_{t}\right) \mapsto\left(C^{-1} M_{1} C, \ldots, C^{-1} M_{t} C\right), \quad C \text { is nonsingular. }
$$

The matrix pair problem also contains the problem of classifying representations of every quiver and every poset (Barot [10] [Section 2.4], Belitskii and Sergeichuk [11], Krause [12] [Section 10]). Moreover, it contains the problem of classifying representations of an arbitrary finite-dimensional algebra (Barot [13] [Proposition 9.14]).

Note that each concrete pair $(A, B)$ of $n \times n$ matrices over an algebraically closed field is reduced to its canonical form $\left(A_{\mathrm{can}}, B_{\mathrm{can}}\right)$ with respect to similarity transformations (1) by Belitskii's algorithm [14] in such a way that $(A, B)$ is similar to $(C, D)$ if and only if $\left(A_{\text {can }}, B_{\text {can }}\right)=\left(C_{\text {can }}, D_{\text {can }}\right)$. However, there is no nonalgorithmic description of the set of Belitskii's canonical pairs under similarity (i.e., the pairs that are not changed by Belitskii's algorithm). Belitskii's algorithm was extended by Sergeichuk [8] to a wide class of matrix problems that includes the problems of classifying representations of quivers and finite dimensional algebras.

The problem of classifying arrays up to equivalence plays the same role in the theory of tensors as the matrix pair problem in the theory of representations of algebras: Futorny, Grochow, and Sergeichuk [15] proved that the problem of classifying three-dimensional arrays up to equivalence transformations contains the problem of classifying every system of tensors of order at most three.

We show that the problem of classifying pairs consisting of a bilinear form and a linear map contains the problem of classifying arbitrary systems of bilinear forms and linear maps.

\section{Main Results}

Many classification problems of linear algebra can be formulated and studied in terms of quiver representations introduced by Gabriel [16]. A quiver is a directed graph. Its representation is given by assigning a vector space to each vertex and a linear map of the corresponding vector spaces to each arrow. This notion plays a central role in the representation theory of finite dimensional algebras since each algebra can be given by a quiver with relations and there is a natural correspondence between their representations; see $[10,13,17-19]$.

Following [20,21], we consider systems of forms and linear maps over a field $\mathbb{F}$ as representations of a mixed graph (i.e., of a graph with undirected and directed edges; multiply edges and loops are allowed): Its vertices represent vector spaces, and its undirected and directed edges represent bilinear forms and linear maps between these spaces. Two representations are isomorphic if these are a set of linear bijections of the corresponding vector spaces that transform one representation into the other; see Definition 1.

Example 1. Consider a mixed graph $Q$ and its representation $\mathcal{R}$ :
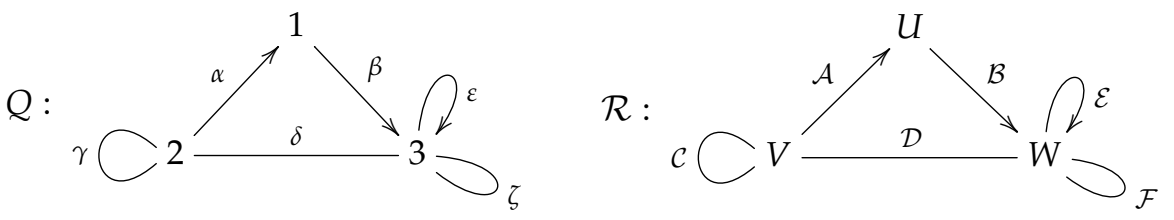

The representation $\mathcal{R}$ consists of vector spaces $U, V, W$ over $\mathbb{F}$, bilinear forms $\mathcal{C}: V \times V \rightarrow \mathbb{F}$, $\mathcal{D}: W \times V \rightarrow \mathbb{F}, \mathcal{F}: W \times W \rightarrow \mathbb{F}$, and linear maps $\mathcal{A}: V \rightarrow U, \mathcal{B}: U \rightarrow W, \mathcal{E}: W \rightarrow W$. 
The vector $\underline{n}=\left(n_{1}, n_{2}, n_{3}\right):=(\operatorname{dim} U, \operatorname{dim} V, \operatorname{dim} W)$ is called the dimension of $\mathcal{R}$. Changing bases in the spaces $U, V, W$, we can reduce the matrices of $\mathcal{R}$ as follows:

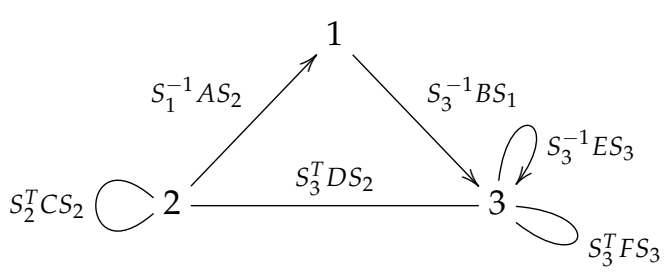

where $S_{1}, S_{2}, S_{3}$ are nonsingular matrices. Thus, the problem of classifying representations of $Q$ of dimension $\underline{n}=\left(n_{1}, n_{2}, n_{3}\right)$ is the problem of classifying all tuples $(A, B, \ldots, F)$ of matrices of sizes $n_{1} \times n_{2}, n_{3} \times n_{1}, \ldots, n_{3} \times n_{3}$ up to these transformations.

For a mixed graph $Q$, we denote by $M_{\underline{n}}(Q)$ the set of representations of dimension $\underline{n}$, in which all vector spaces are of the form $\mathbb{F}^{k}$ with $k=0,1,2, \ldots$ The set $M_{\underline{n}}(Q)$ is a vector space over $\mathbb{F}$; its elements are matrix tuples (see Definition 2 ). We say that the problem of classifying representations of a mixed graph $Q$ is contained in the problem of classifying representations of a mixed graph $Q^{\prime}$ if for each dimension $\underline{n}$ there exists an affine injection (That is, $F(A)=R+\varphi(A)$ for $R \in M_{\underline{n}^{\prime}}\left(Q^{\prime}\right)$ and all $A \in \bar{M}_{\underline{n}}(Q)$, in which $\varphi: M_{\underline{n}}(Q) \rightarrow M_{\underline{n}^{\prime}}\left(Q^{\prime}\right)$ is a linear injection).

$$
F: M_{\underline{n}}(Q) \rightarrow M_{\underline{n^{\prime}}}\left(Q^{\prime}\right)
$$

of a very special type given in Definition 3 such that $A, B \in M_{n}(Q)$ are isomorphic if and only if $F(A), F(B) \in M_{\underline{n}^{\prime}}\left(Q^{\prime}\right)$ are isomorphic.

The problem of classifying representations of $Q$ and $Q^{\prime}$ are equivalent if each of these problems contains the other.

The main result of this paper is the following theorem, which is proved in Sections 4 and 5.

\section{Theorem 1.}

(a) The problem of classifying representations of $\subset \bullet$ (i.e., of pairs consisting of a bilinear form and a linear map) contains the problem of classifying representations of each mixed graph.

(b) Let a mixed graph $Q$ satisfy the following condition:

$Q$ contains a cycle in which the number of undirected edges is odd, and $Q$ contains an edge outside of this cycle but with a vertex (or both the vertices) in this cycle.

Then the problem of classifying representations of $Q$ is equivalent to the problem of classifying representations of $\subset \cdot \supset$.

Let us derive a corollary of Theorem 1.

Let $Q$ be a connected mixed graph that does not satisfy the condition (4). The representations of mixed graphs that are cycles are classified in [20] [\$3]. Suppose that $Q$ does not contain a cycle in which the number of undirected edges is odd; in particular, it does not contain undirected loops. Then $Q$ can be transformed to a quiver $\bar{Q}$ with the same underlying graph (obtained by deleting the orientation of the edges) using the following procedure described in [20]: 
If $v$ is a vertex of $Q$, then we denote by $Q^{v}$ the graph that is obtained from $Q$ by deleting (resp., adding) the arrows at the ends of all edges in the vertex $v$ that have it (resp., do not have it); we say that $Q^{v}$ is obtained from $Q$ by dualization at $v$. For example:
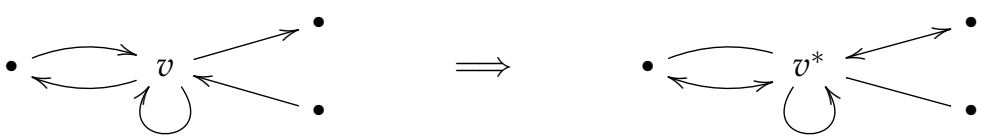

(we write $v^{*}$ instead of $v$ ). Thus, $v \longrightarrow w$ is replaced by $v^{*} \leftrightarrow w$. Then we replace $w$ with $w^{*}$ and obtain $v^{*} \longleftarrow w^{*}$. Since $Q$ does not contain a cycle in which the number of undirected edges is odd, we can reduce $Q$ to some quiver $\bar{Q}$ by these replacements.

There is a natural correspondence between the representations of $Q$ and $Q^{v}:$ If $\mathcal{A}$ is a representation of $Q$, then the representation $\mathcal{A}^{v}$ of $Q^{v}$ is obtained by replacing the vector space $V$ assigned to $v$ with the dual space $V^{*}$ of all linear forms on $V$. This correspondence is based on the fact that each bilinear form $B: V \times U \rightarrow \mathbb{F}$ defines the linear map $U \rightarrow V^{*}$ via $x \mapsto B(?, x)$, and each linear map $\mathcal{A}: U \rightarrow V$ defines the bilinear form $V^{*} \times U \rightarrow \mathbb{F}$ via $(x *, y) \mapsto x^{*} A y$.

Therefore, the theory of representations of mixed graphs without cycles in which the number of undirected edges is odd is the theory of quiver representations. The representation types of quivers are well known; the representations of tame quivers are classified by Donovan and Freislich [4], and Nazarova [22]. The other quivers are wild; the problem of classifying representations of each wild quiver is equivalent to the problem of classifying representations of $C \cdot \supset$ (i.e., of matrix pairs up to similarity transformations (1)); see [10-12] and Lemma 1.

We say that a mixed graph $Q$ is tame if it is reduced by dualizations at vertices to the disjoint union of mixed cycles and a tame quiver (thus, the classification of representations of tame mixed graphs is known). A mixed graph $Q$ is wild if it is reduced by dualizations at vertices to a wild quiver. A mixed graph $Q$ is superwild if the problem of classifying its representations is equivalent to the problem of classifying representations of $\subset \cdot D$.

Corollary 1. Each mixed graph is tame, wild, or superwild.

(i) A mixed graph is tame if its underlying graph is the disjoint union of some copies of the Dynkin diagrams and the extended Dynkin diagrams

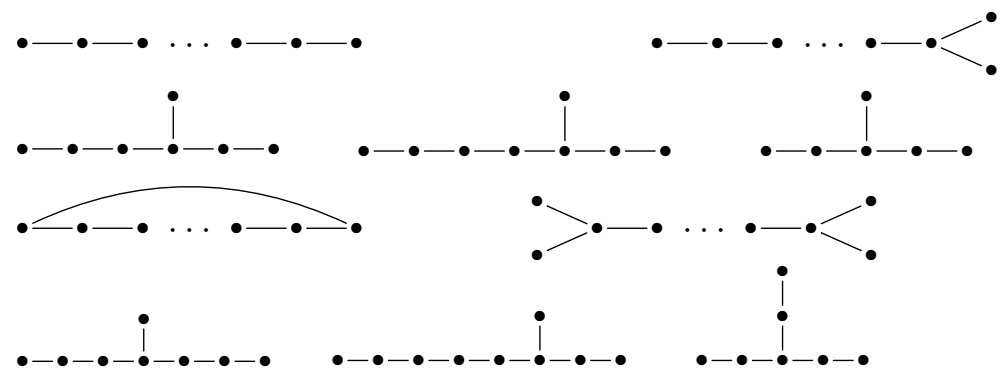

(ii) A mixed graph is wild if it is not tame and it does not contain a cycle in which the number of undirected edges is odd.

(iii) A mixed graph is superwild if it satisfies condition (4).

Proof. Let $Q$ be a mixed graph. If (4) holds, then $Q$ is superwild by Theorem 1(c). Suppose that (4) does not hold. Then $Q$ is the disjoint union of mixed cycles and a mixed graph $Q_{0}$ without a cycle in which the number of undirected edges is odd. The graph $Q_{0}$ is reduced to a quiver $\bar{Q}_{0}$ by dualizations at vertices. By definition, $Q$ is tame or wild if and only if $\bar{Q}_{0}$ is tame or wild, respectively. By $[4,22], \bar{Q}_{0}$ is tame if and only if its underlying graph is the disjoint union of some copies of graphs (5). 


\section{The Category of Representations}

The category of representations of a mixed graph (in general, nonadditive) is defined as follows.

Definition 1 ([21]; see also [18]). Let $Q$ be a mixed graph with vertices $1, \ldots, t$. Its representation $\mathcal{A}$ over a field $\mathbb{F}$ is given by assigning a vector space $\mathcal{A}_{v}$ over $\mathbb{F}$ to each vertex $v$, a bilinear form $\mathcal{A}_{\alpha}: \mathcal{A}_{v} \times \mathcal{A}_{u} \rightarrow \mathbb{F}$ to each undirected edge $\alpha: u \_v$ with $u \leqslant v$ (this inequality is given for uniqueness), and a linear map $\mathcal{A}_{\beta}: \mathcal{A}_{u} \rightarrow \mathcal{A}_{v}$ to each directed edge $\beta: u \longrightarrow v$. A morphism $\varphi: \mathcal{A} \rightarrow \mathcal{B}$ of two representations of $Q$ is a family of linear maps $\varphi_{1}: \mathcal{A}_{1} \rightarrow \mathcal{B}_{1}, \ldots, \varphi_{t}: \mathcal{A}_{t} \rightarrow \mathcal{B}_{t}$ such that $\mathcal{A}_{\alpha}(y, x)=\mathcal{B}_{\alpha}\left(\varphi_{v} y, \varphi_{u} x\right)$ for each $\alpha: u$ — with $u \leqslant v$, and $\varphi_{v} \mathcal{A}_{\beta}=\mathcal{B}_{\beta} \varphi_{u}$ for each $\beta: u \longrightarrow v$.

In the following definition, we consider representations, in which all vector spaces are $\mathbb{F}^{k}$ with $k=0,1,2, \ldots$ Such representations are given by their matrices, as in (3), and so we call them "matrix representations". The category $M(Q)$ of matrix representations is defined as follows.

Definition 2. Let $Q$ be a mixed graph with vertices $1, \ldots, t$. A matrix representation $A$ of dimension $\underline{n}=\left(n_{1}, \ldots, n_{t}\right)$ of $Q$ over a field $\mathbb{F}$ is given by assigning a matrix $A_{\alpha} \in \mathbb{F}^{n_{v} \times n_{u}}$ to each undirected edge $\alpha: u-v$ with $u \leqslant v$ and to each directed edge $\alpha: u \longrightarrow v$. A morphism $\varphi: A \rightarrow B$ of matrix representations of dimensions $\underline{n}$ and $\underline{n}^{\prime}$ is a family of matrices $S_{1}, \ldots, S_{t}$ of sizes $n_{1}^{\prime} \times n_{1}, \ldots, n_{t}^{\prime} \times n_{t}$ such that

$$
A_{\alpha}=S_{v}^{T} B_{\alpha} S_{u}, \quad S_{v} A_{\beta}=B_{\beta} S_{u}
$$

for each undirected edge $\alpha: u-v(u \leqslant v)$ and each directed edge $\beta: u \longrightarrow v$.

The set $M_{\underline{n}}(Q)$ of matrix representations of dimension $\underline{n}$ is a vector space over $\mathbb{F}$.

Definition 3. Let $Q$ and $Q^{\prime}$ be mixed graphs with vertices $1, \ldots, t$ and $1, \ldots, t^{\prime}$, and with edges $\alpha_{1}, \ldots, \alpha_{p}$ and $\beta_{1}, \ldots, \beta_{q}$, respectively. The problem of classifying representations of $Q$ is contained in the problem of classifying representations of $Q^{\prime}$, we write $Q \lesssim Q^{\prime}$, if there exists a functor

$$
\mathcal{F}: M(Q) \rightarrow M\left(Q^{\prime}\right)
$$

with the following properties:

- $\quad \mathcal{F}$ is injective on objects; moreover, for each $\underline{n}=\left(n_{1}, \ldots, n_{t}\right)$ there exists $\underline{n}^{\prime}=\left(n_{1}^{\prime}, \ldots, n_{t^{\prime}}^{\prime}\right)$ (all $n_{i}$ and $n_{j}^{\prime}$ are nonnegative integers) such that $\mathcal{F}$ maps $M_{\underline{n}}(Q)$ to $M_{\underline{n}^{\prime}}\left(Q^{\prime}\right)$ and this map is an affine injection of vector spaces.

- $\mathcal{F}$ is injective on morphisms; moreover, $A, B \in M_{\underline{n}}(Q)$ are isomorphic if and only if $\mathcal{F}(A), \mathcal{F}(B) \in M_{\underline{n}^{\prime}}\left(Q^{\prime}\right)$ are isomorphic.

- $\mathcal{F}$ is produced by a parameter matrix representation of $Q^{\prime}$ (compare with [7] [p. 338]), which means that there exists a parameter matrix representation

$$
N(x):=\left(N_{\beta_{1}}(x), \ldots, N_{\beta_{q}}(x)\right)
$$

of $Q^{\prime}$ with parameters $x_{1}, x_{2}, \ldots, x_{p}$ such that each $x_{i}$ is an exactly one entry of an exactly one matrix among $N_{\beta_{1}}(x), \ldots, N_{\beta_{q}}(x)$, the other of their entries are elements of $\mathbb{F}$, and

$$
\mathcal{F}(A)=\left(N_{\beta_{1}}(A), \ldots, N_{\beta_{q}}(A)\right)
$$

for each matrix representation $A=\left(A_{\alpha_{1}}, \ldots, A_{\alpha_{p}}\right)$ of $Q$. The representation (7) of $Q^{\prime}$ is constructed on (6) as follows:

- Rearranging the basis vectors in the vector spaces of the representation $N(x)$, we rearrange the rows and columns of its matrices converting $N(0)$ to a direct sum 
$M_{1} \oplus \cdots \oplus M_{r}$ of matrix representations of $Q^{\prime}$ of nonzero dimension with the maximum number $r$. We say that two rows or columns of $N(x)$ are lined if they (with $x=0$ ) are converted to rows or columns from the same summand $M_{i}$. Thus, there are rclasses $\mathcal{L}_{1}, \ldots, \mathcal{L}_{r}$ of linked rows and columns; we require that each class contains a row or column with a parameter.

- $\quad$ Let $n_{1}, \ldots, n_{r}$ be natural numbers. Denote by $N\left(K_{1}, \ldots, K_{t}\right)$ a matrix representation of $Q^{\prime}$ obtained from $N(x)$ by replacing all rows and columns that belong to the same class $\mathcal{L}_{j}$ by strips of size $n_{j}$ such that each parameter $x_{i}$ is replaced by an arbitrary matrix $K_{i}$ of suitable size, each entry that is a nonzero element $\alpha \in \mathbb{F}$ is replaced by $\alpha I$, and each zero entry is replaced by the zero block.

Two mixed graphs $Q$ and $Q^{\prime}$ are equivalent if $Q \lesssim Q^{\prime}$ and $Q^{\prime} \lesssim Q$ (see Definition 3).

Example 2. The problem of classifying representations of the quiver $C \bullet P$ is contained in the problem of classifying representations of $C \bullet \longleftarrow \bullet$ (i.e., $\subset \bullet \supset \lesssim C \bullet \longleftarrow \bullet$ ) via

$$
\left(N_{1}(x), N_{2}(x)\right):=\left(\left[\begin{array}{ll}
0 & x_{1} \\
1 & x_{2}
\end{array}\right],\left[\begin{array}{l}
1 \\
0
\end{array}\right]\right)
$$

since

- $\quad$ each morphism $\varphi: A \subset \bullet \bigcirc B \rightarrow A^{\prime} \subset \bullet \bigcirc B^{\prime}$ (where $A, B \in \mathbb{F}^{n \times n}$ and $A^{\prime}, B^{\prime} \in$ $\mathbb{F}^{n^{\prime} \times n^{\prime}}$ ) is given by a matrix $S \in \mathbb{F}^{n^{\prime} \times n}$ such that $S A=A^{\prime} S$ and $S B=B^{\prime} S$; the corresponding morphism $\mathcal{F}(\varphi): \mathcal{F}(A, B) \rightarrow \mathcal{F}\left(A^{\prime}, B^{\prime}\right)$, where

$$
\mathcal{F}(A, B)=\left(\left[\begin{array}{ll}
0_{n} & A \\
I_{n} & B
\end{array}\right],\left[\begin{array}{c}
I_{n} \\
0_{n}
\end{array}\right]\right), \quad \mathcal{F}\left(A^{\prime}, B^{\prime}\right)=\left(\left[\begin{array}{cc}
0_{n^{\prime}} & A^{\prime} \\
I_{n^{\prime}} & B^{\prime}
\end{array}\right],\left[\begin{array}{l}
I_{n^{\prime}} \\
0_{n^{\prime}}
\end{array}\right]\right)
$$

is given by the matrix pair $(S \oplus S, S)$;

- $\quad(A, B)$ is reduced to $\left(A^{\prime}, B^{\prime}\right)$ by similarity transformations (1) if and only if $\mathcal{F}(A, B)$ is reduced to $\mathcal{F}\left(A^{\prime}, B^{\prime}\right)$ by transformations

$$
\left(S^{-1}\left[\begin{array}{cc}
0_{n} & A \\
I_{n} & B
\end{array}\right] S, S^{-1}\left[\begin{array}{c}
I_{n} \\
0_{n}
\end{array}\right] R\right), \quad S \text { and } R \text { are nonsingular. }
$$

\section{Proof of Theorem 1(a)}

Let us consider a matrix representation

$$
H \subset \bullet \supset J, \quad J:=J_{k_{1}}\left(0_{n_{1}}\right) \oplus \cdots \oplus J_{k_{\tau}}\left(0_{n_{\tau}}\right),
$$

in which

$$
J_{k_{i}}\left(0_{n_{i}}\right):=\left[\begin{array}{cccc}
0_{n_{i}} & I_{n_{i}} & & 0 \\
& 0_{n_{i}} & \ddots & \\
& & \ddots & I_{n_{i}} \\
0 & & & 0_{n_{i}}
\end{array}\right] \quad\left(k_{i} \text { diagonal blocks }\right) .
$$

We reduce $H \subset \bullet \supset J$ by those admissible transformations that preserve $J:$

$$
(H, J) \mapsto\left(S^{T} H S, S^{-1} J S\right), \quad S \text { is nonsingular and } S^{-1} J S=J .
$$


Partition $S=\left[S_{i j}\right]_{i, j=1}^{\tau}$ conformally to $J$. Since $J S=S J$, every $S_{i j}$ has the form

$$
\left[\begin{array}{cccccc}
C_{i j} & C_{i j}^{\prime} & C_{i j}^{\prime \prime} & C_{i j}^{\prime \prime \prime} & \ddots \\
& & C_{i j} & C_{i j}^{\prime} & C_{i j}^{\prime \prime} & \ddots \\
& & & C_{i j} & C_{i j}^{\prime} & \ddots \\
& & & & C_{i j} & \ddots \\
0 & & & & & \ddots
\end{array}\right] \text { or }\left[\begin{array}{lllll}
C_{i j} & C_{i j}^{\prime} & C_{i j}^{\prime \prime} & C_{i j}^{\prime \prime \prime} & \ddots \\
& C_{i j} & C_{i j}^{\prime} & C_{i j}^{\prime \prime} & \ddots \\
& & C_{i j} & C_{i j}^{\prime} & \ddots \\
& & & C_{i j} & \ddots \\
& & & & \ddots \\
& & & & \\
0 & & & &
\end{array}\right] \text {, }
$$

where all $C_{i j}$ are arbitrary $n_{i} \times n_{j}$ matrices such that $S$ is nonsingular. Replacing all offdiagonal blocks of $S$ by zeros, we obtain the block diagonal matrix

$$
S_{\text {diag }}:=(\underbrace{S_{1} \oplus S_{1} \oplus S_{1} \oplus \cdots}_{k_{1} \text { summands }}) \oplus \cdots \oplus(\underbrace{S_{\tau} \oplus S_{\tau} \oplus S_{\tau} \oplus \cdots}_{k_{\tau} \text { summands }}),
$$

in which every $S_{i}:=C_{i i}$ is $n_{i} \times n_{i}$.

By Belitskii's theorem (see [23] [Section 3.4] or [8] [Theorem 1.2]), the Jordan matrix J is permutation similar to a nilpotent Weyr matrix such that all matrices commuting with it are upper block triangular. Since $J S=S J$, Belitskii's theorem ensures that the matrix $S$ is permutation similar to a block triangular matrix whose main block diagonal coincides with the sequence of summands in (10), up to permutation of these summands. Hence, $S$ is nonsingular if and only if all blocks $S_{1}, \ldots, S_{\tau}$ are nonsingular. Moreover, if the matrix $H$ in (8) is partitioned such that the sizes of its diagonal blocks coincide with the sizes of the direct summands in (10), then

$$
\begin{aligned}
& \text { if each horizontal strip and each vertical strip of } H \text { contains } \\
& \text { at most one nonzero block, then } S^{T} H S=S_{\text {diag }}^{T} H S_{\text {diag. }} \text {. }
\end{aligned}
$$

Let us prove Theorem 1(a) for the mixed graph $Q$ in (2); its proof for an arbitrary mixed graph is analogous. Let
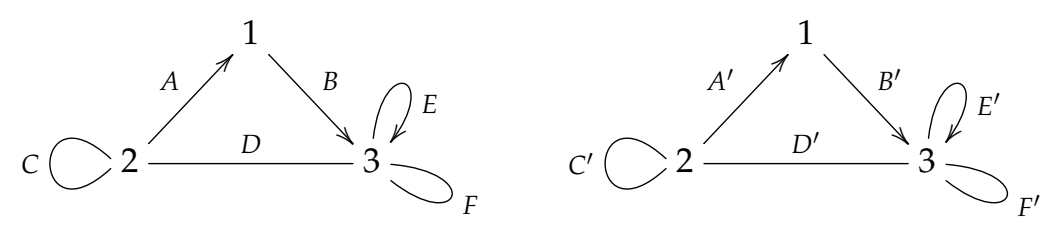

be matrix representations of $Q$ of dimension $\left(n_{1}, n_{2}, n_{3}\right)$. We construct the matrix represen- 
tations $H \subset \bullet \supset J$ and $H^{\prime} \subset \bullet \supset J$ as follows:

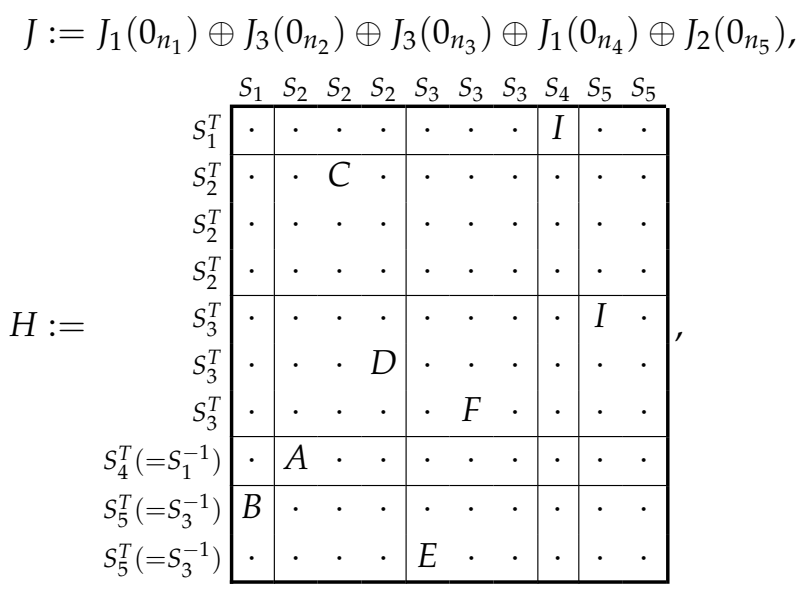

in which the points denote zero blocks; the matrix $H^{\prime}$ is obtained from $H$ by replacing $A, B, \ldots, F$ with $A^{\prime}, B^{\prime}, \ldots, F^{\prime}$.

If $J S=S J$, then

$$
S_{\text {diag }}=S_{1} \oplus S_{2} \oplus S_{2} \oplus S_{2} \oplus S_{3} \oplus S_{3} \oplus S_{3} \oplus S_{4} \oplus S_{5} \oplus S_{5}
$$

(see (10)). The summands of (15) are written in (14) over the vertical strips of $H$, and their transposes are written to the left of the horizontal strips of $H$. By (11), each nonzero block of (14) is multiplied by them if $(H, J)$ is reduced by transformations (9).

We must prove that $(H, J)$ is reduced to $\left(H^{\prime}, J\right)$ by transformations (9) if and only if the representations (12) are isomorphic; that is, if and only if $A, B, \ldots, F$ are reduced to $A^{\prime}, B^{\prime}, \ldots, F^{\prime}$ by transformations (3).

Let $(H, J)$ be reduced to $\left(H^{\prime}, J\right)$ by transformations (9). By (11),

$$
\begin{aligned}
& S_{1}^{T} I S_{4}=I, \quad S_{3}^{T} I S_{5}=I, \quad S_{4}^{T} A S_{2}=A^{\prime}, \quad S_{5}^{T} B S_{1}=B^{\prime}, \\
& S_{2}^{T} C S_{2}=C^{\prime}, \quad S_{3}^{T} D S_{2}=D^{\prime}, \quad S_{5}^{T} E S_{3}=E^{\prime}, \quad S_{3}^{T} F S_{3}=F^{\prime} \text {. }
\end{aligned}
$$

The first two equalities ensure that $S_{4}^{T}=S_{1}^{-1}$ and $S_{5}^{T}=S_{3}^{-1}$. Therefore, $A, B, \ldots, F$ are reduced to $A^{\prime}, B^{\prime}, \ldots, F^{\prime}$ by transformations (3).

Conversely, let $A, B, \ldots, F$ be reduced to $A^{\prime}, B^{\prime}, \ldots, F^{\prime}$ by transformations (3). Then $(H, J)$ is reduced to $\left(H^{\prime}, J\right)$ by transformations (9) with $S$ of the form (15), in which $S_{4}:=$ $S_{1}^{-T}$ and $S_{5}:=S_{3}^{-T}$.

This completes the proof of Theorem 1(a). The following lemma is proved analogously; it is also proved in [10] [Section 2.4], [11], and [12] [Section 10].

Lemma 1. The problem of classifying representations of $C \bullet$ contains the problem of classifying representations of each quiver.

Proof. Let $Q$ be a quiver, and let $A$ be its matrix representation. We construct a matrix representation $H \subset \cdot \supset J$ as follows.

The matrix $J$ is given in (8). The equality $S^{-1} J S=J$ implies that the main block diagonal of $S$ is (10).

We take $H$ in which each horizontal strip and each vertical strip contains at most one nonzero block. By analogy with (11), $H$ is reduced by transformations $S_{\text {diag }}^{-1} H S_{\text {diag. }}$. We construct $H$ such that some of its blocks are the matrices of $A$ and they are reduced by the same transformations as in $A$, and the other blocks are zero. 


\section{Proof of Theorem 1(b)}

Lemma 2. Let a connected mixed graph $Q$ contain a cycle in which the number of undirected edges is odd, and let $Q$ not coincide with this cycle. Then the problem of classifying its representations contains the problem of classifying representations of one of the mixed graphs

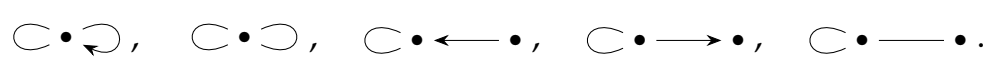

Proof. Let a connected mixed graph $Q$ contain a cycle $\mathcal{C}$ in which the number of undirected edges is odd, and let $\alpha$ be an edge outside of $\mathcal{C}$ with vertices $v_{1} \in \mathcal{C}$ and $u$.

Case 1: $u=v_{1}$. Then $Q$ contains a subgraph

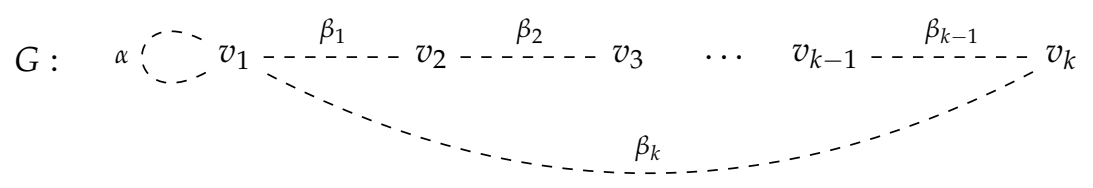

in which $k \geqslant 1$ and each dotted line is an undirected or directed edge.

Consider the matrix representation

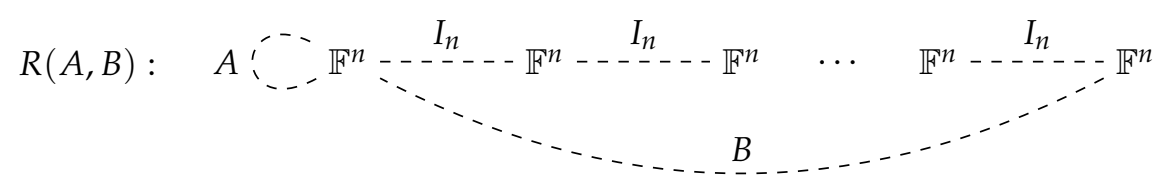

of $Q$, in which $A, B \in \mathbb{F}^{n \times n}$ and all vertices outside of $\mathcal{C}$ are assigned by the zero spaces. Let us prove that :- $\bigcirc \lesssim Q$ with a suitable direction of the left loop in $-\supset$. We need to show the following equivalence of isomorphisms:

$$
A: \bullet \bigcirc B \simeq A^{\prime}: \cdot \bigcirc B^{\prime} \Longleftrightarrow R(A, B) \simeq R\left(A^{\prime}, B^{\prime}\right)
$$

(" $\simeq$ " means "is isomorphic to").

Let us prove " $\Longleftarrow^{\prime}$. Let $R(A, B) \simeq R\left(A^{\prime}, B^{\prime}\right)$ via $\left(S_{1}, \ldots, S_{k}\right)$. Then $S_{1}=S_{2}^{-T}$ if $\alpha_{1}$ is undirected and $S_{1}=S_{2}$ if $\alpha_{1}$ is directed. Analogously, $S_{2}=S_{3}^{-T}$ if $\alpha_{2}$ is undirected and $S_{2}=S_{3}$ if $\alpha_{2}$ is directed, and so on. Since the number of undirected edges of $\mathcal{C}$ is odd, $S_{k-1}=S_{k}$ if $\alpha_{k}$ is undirected and $S_{k-1}=S_{k}^{-T}$ if $\alpha_{k}$ is directed. Hence, $B^{\prime}=S_{1}^{T} B S_{1}$, and so $A: \bullet \bigcirc \simeq A^{\prime}: \bullet B^{\prime}$ via $S_{1}$, in which the left loop in $: \bullet \bigcirc$ is directed as the left loop in (17). The implication " $\Longrightarrow$ " is proved analogously.

Case 2: $u=v_{r}$ with $r \neq 1$. If $\alpha: v_{1} \longleftarrow v_{r}$, then we take $v_{r}$ as $v_{1}$. We have that $\alpha: v_{1} \longrightarrow v_{r}$ or $\alpha: v_{1} \longrightarrow v_{r}$, and so $Q$ contains the subgraph $G_{r}$ that is obtained from (17) by replacing $\alpha:-\bullet$ with $\alpha: v_{1}--v_{r}$.

Denote by $R_{r}(A, B)$ the matrix representation of $G_{r}$ that is obtained from (18) by deleting $A:-\bullet$ and assigning $A$ to $\alpha: v_{1}-v_{r}$. Let us prove that $\bullet \supset \lesssim Q$, in which the left loop is directed if and only if

- $\alpha$ in (17) is directed and the number of undirected edges in $\beta_{1}, \ldots \beta_{r-1}$ is even, or

- $\alpha$ in (17) is undirected and the number of undirected edges in $\beta_{1}, \ldots \beta_{r-1}$ is odd.

This statement holds since

$$
A: \cdot \bigcirc B \simeq A^{\prime}: \cdot \bigcirc B^{\prime} \Longleftrightarrow R_{r}(A, B) \simeq R_{r}\left(A^{\prime}, B^{\prime}\right),
$$

which is proved in (19). 
Case 3: $u \notin \mathcal{C}$. Then $Q$ contains the subgraph $G^{\prime}$ that is obtained from (17) by replacing $\alpha:=-$ with $\alpha: u--v_{1}$. Denote by $R^{\prime}(A, B)$ the matrix representation of $G^{\prime}$ that is obtained from (18) by deleting $A,: \bullet$ and assigning $A$ to $\alpha: u--v_{1}$. Since

$$
\text { • } A \supset \supset \simeq A^{\prime} \cdot{ }^{\prime} \Longleftrightarrow B^{\prime}(A, B) \simeq R^{\prime}\left(A^{\prime}, B^{\prime}\right) \text {, }
$$

we have $\cdot-\cdots \cdot \lesssim Q$.

Lemma 3. $\subset \cdot \supset \lesssim C \cdot \supset$.

Proof. Write

$$
A:=\left[\begin{array}{cc}
X & 0 \\
Y & 0
\end{array}\right], \quad A^{\prime}:=\left[\begin{array}{cc}
X^{\prime} & 0 \\
Y^{\prime} & 0
\end{array}\right], \quad B:=\left[\begin{array}{ll}
0 & I \\
0 & 0
\end{array}\right],
$$

in which all blocks are $n \times n$. We need to show the following equivalence of isomorphisms:

$$
X \subset \bullet \supset Y \simeq X^{\prime} \subset \bullet \supset Y^{\prime} \Longleftrightarrow A \subset \cdot \supset B \simeq A^{\prime} \subset \cdot \supset B
$$

$\Longleftarrow$. Let the right isomorphism hold via $S$. The equality $S^{T} B S=B$ takes the form

$$
\left[\begin{array}{ll}
S_{1}^{T} S_{3} & S_{1}^{T} S_{4} \\
S_{2}^{T} S_{3} & S_{2}^{T} S_{4}
\end{array}\right]=\left[\begin{array}{ll}
0 & I \\
0 & 0
\end{array}\right], \quad \text { in which } S=\left[\begin{array}{ll}
S_{1} & S_{2} \\
S_{3} & S_{4}
\end{array}\right] .
$$

Therefore, $S_{1}^{T} S_{4}=I, S_{2}=S_{3}=0$, and $S=S_{1} \oplus S_{1}^{-T}$. Hence, the left isomorphism in (20) holds via $S_{1}$.

$C \oplus \overrightarrow{C^{-T}}$. If the left isomorphism holds via $C$, then the right isomorphism holds via

Lemma 4. $\subset \cdot \supset \lesssim \subset \cdot \longleftarrow \cdot$

Proof. Due to Lemma 3, it is sufficient to prove that $\subset \bullet \supset \lesssim \subset \bullet \longleftarrow \bullet$. Write

$$
A:=\left[\begin{array}{ccc}
0 & I & 0 \\
0 & 0 & I \\
X & 0 & Y
\end{array}\right], \quad A^{\prime}:=\left[\begin{array}{ccc}
0 & I & 0 \\
0 & 0 & I \\
X^{\prime} & 0 & Y^{\prime}
\end{array}\right], \quad B:=\left[\begin{array}{ll}
I & 0 \\
0 & I \\
0 & 0
\end{array}\right],
$$

in which all blocks are $n \times n$. We need to show the following equivalence of isomorphisms:

$$
X \subset \bullet \supset Y \simeq X^{\prime} \subset \bullet \supset Y^{\prime} \Longleftrightarrow A \subset \bullet \stackrel{B}{\longleftarrow} \simeq A^{\prime} \subset \bullet \stackrel{B}{\longleftarrow}
$$

$\Longleftarrow$. Let the right isomorphism hold. Then

$$
\left(S^{T} A S, S^{-1} B R\right)=\left(A^{\prime}, B\right) \quad \text { for nonsingular } S \text { and } R .
$$

The equality $B R=S B$ implies that $S$ and $R$ have the form

$$
S=\left[\begin{array}{ccc}
S_{1} & S_{2} & S_{3} \\
S_{4} & S_{5} & S_{6} \\
0 & 0 & S_{7}
\end{array}\right], \quad R=\left[\begin{array}{ll}
S_{1} & S_{2} \\
S_{4} & S_{5}
\end{array}\right] .
$$

Substituting them to $S^{T} A S=A^{\prime}$, we obtain

$$
\left[\begin{array}{ccc}
S_{1}^{T} S_{4} & S_{1}^{T} S_{5} & S_{1}^{T} S_{6}+S_{4}^{T} S_{7} \\
S_{2}^{T} S_{4} & S_{2}^{T} S_{5} & S_{2}^{T} S_{6}+S_{5}^{T} S_{7} \\
S_{3}^{T} S_{4}+S_{7}^{T} X S_{1} & S_{3}^{T} S_{5}+S_{7}^{T} X S_{2} & S_{3}^{T} S_{6}+S_{6}^{T} S_{7}+S_{7}^{T} X S_{3}+S_{7}^{T} Y S_{7}
\end{array}\right]=A^{\prime} .
$$


Since the rows of $R$ are linearly independent, $S_{2}^{T}\left[S_{4} S_{5}\right]=[00]$ implies $S_{2}=0$. Since $S_{1}^{T} S_{5}=I, S_{1}^{T} S_{4}=0$ implies $S_{4}=0$. Equating the $(2,3)$ blocks gives $S_{5}^{T} S_{7}=I$. Equating the $(1,3)$ blocks gives $S_{6}=0$. Equating the last horizontal strips gives

$$
\left[\begin{array}{lll}
S_{7}^{T} X S_{1} & S_{3}^{T} S_{5} & S_{7}^{T} X S_{3}+S_{7}^{T} Y S_{7}
\end{array}\right]=\left[\begin{array}{lll}
X^{\prime} & 0 & Y^{\prime}
\end{array}\right] .
$$

Since $S_{3}^{T} S_{5}=0$ and $S_{5}$ is nonsingular, we have $S_{3}=0$. Therefore, $S=S_{1} \oplus S_{1}^{-T} \oplus S_{1}$, and so $S_{1}^{T} X S_{1}=X^{\prime}$ and $S_{1}^{T} Y S_{1}=Y^{\prime}$.

$\Longrightarrow$. If the left isomorphism holds via $C$, then (23) holds for $S=C \oplus C^{-T} \oplus C$ and $R=C \oplus C^{-T}$.

Lemma 5. $\subset \cdot \supset \lesssim C \cdot \longrightarrow \cdot$ and $C \cdot \supset \lesssim C \cdot-\cdot$

Proof. It is sufficient to prove that $\subset \bullet \supset \lesssim \subset \bullet \longrightarrow \bullet$ and $\subset \bullet \supset \lesssim \subset \bullet-\bullet$ because of Lemma 3. We take $A$ and $A^{\prime}$ as in (21) and $B=\left[\begin{array}{lll}0 & 0 & I\end{array}\right]$. We must prove (22), in which $\longleftarrow$ is replaced by $\longrightarrow$ and by $\_$. The proof is the same since the equality $B S=R B$ implies that $S$ as in (24).

Proof of Theorem 1(b). Let a mixed graph $Q$ satisfy the condition (4). By Theorem 1(a), $Q \lesssim C \bullet \supset$. Lemma 2 ensures that $H \lesssim Q$, in which $H$ is one of the mixed graphs (16). By Lemmas $3-5, \subset \bullet \supset \lesssim H$. Hence, $\subset \bullet \supset \lesssim Q$.

\section{Conclusions}

We have proved that the problem of classifying matrix pairs with respect to transformations

$$
(A, B) \mapsto\left(S^{T} A S, S^{-1} B S\right), \quad S \text { is nonsingular }
$$

contains the problem of classifying an arbitrary system of bilinear forms and linear maps. There are only two essentially different unsolved classification problems for systems of forms and linear maps: The classical unsolved problem about matrix pairs under similarity and the problem of classifying matrix pairs under transformations (25). These problems are given by the graphs $C \bullet \supset$ and $\subset \bullet \supset$. There is no sense in studying representations of each of these graphs (and of any graph that is equivalent to one of them) outside the general theory of representations of quivers or mixed graphs, respectively. Likewise, Belitskii's algorithm that was constructed for matrix pairs under similarity can be applied to matrices of an arbitrary system of linear maps.

Author Contributions: Formal analysis, C.M.d.F.; Investigation, A.A. and V.F.; Methodology, M.A.; Project administration, M.A.; Writing—original draft, V.V.S.; Writing—review \& editing, C.M.d.F. The authors contributed equally to this work. All authors have read and agreed to the published version of the manuscript.

Funding: This work was supported and funded by Kuwait University, Research Grant No. SM01/19.

Institutional Review Board Statement: Not applicable.

Informed Consent Statement: Not applicable.

Data Availability Statement: Not applicable.

Conflicts of Interest: The authors declare no conflict of interest.

\section{References}

1. Bašev, V.A. Representations of the group $Z_{2} \times Z_{2}$ in a field of characteristic 2. (Russian). Dokl. Akad. Nauk SSSR 1961, 141, 1015-1018.

2. Brenner, S. Modular representation of p-group. J. Algebra 1970, 15, 89-102. [CrossRef]

3. Krugljak, S.A. Representations of the group $(p, p)$ over a field of characteristic $p$. (Russian). Dokl. Akad. Nauk SSSR 1963, 153, 1253-1256. 
4. Donovan, P.; Freislich, M.R. Some evidence for an extension of the Brauer-Thrall conjecture. Sonderforschungsbereich Theor. Math. 1972, 40, 24-26.

5. Drozd, Y.A. Tame and wild matrix problems. Lect. Notes in Math. 1980, 832, 242-258.

6. Crawley-Boevey, W.W. On tame algebras and bocses. Proc. London Math. Soc. 1988, 56, 451-483. [CrossRef]

7. Gabriel, P.; Nazarova, L.A.; Roiter, A.V.; Sergeichuk, V.V.; Vossieck, D. Tame and wild subspace problems. Ukr. Math. J. 1993, 45, 335-372. [CrossRef]

8. Sergeichuk, V.V. Canonical matrices for linear matrix problems. Linear Algebra Appl. 2000, 317, 53-102. [CrossRef]

9. Gelfand, I.M.; Ponomarev, V.A. Remarks on the classification of a pair of commuting linear transformations in a finite dimensional vector space. Functional Anal. Appl. 1969, 3, 325-326. [CrossRef]

10. Barot, M. Representations of Quivers. Notes for a Course at the "Advanced School on Representation Theory and Related Topics"; ICTP: Trieste, Italy, 2006. Available online: matem.unam.mx/barot/articles/notes_ictp.pdf (accessed on 4 February 2021).

11. Belitskii, G.R.; Sergeichuk, V.V. Complexity of matrix problems. Linear Algebra Appl. 2003, 361, 203-222. [CrossRef]

12. Krause, H. Representations of Quivers via Reflection Functors. arXiv 2008, arXiv:0804.1428.

13. Barot, M. Introduction to the Representation Theory of Algebras; Springer: Cham, Switzerland, 2015.

14. Belitskiı̌, G.R. Normal forms in a space of matrices. In Analysis in Infinite-Dimensional Spaces and Operator Theory; Marchenko, V.A., Ed.; Naukova Dumka: Kiev, Ukraine, 1983; pp. 3-15. (In Russian)

15. Futorny, V.; Grochow, J.A.; Sergeichuk, V.V. Wildness for tensors. Linear Algebra Appl. 2019, 566, 212-244. [CrossRef]

16. Gabriel, P. Unzerlegbare Darstellungen I. Manuscripta Math. 1972, 6, 71-103. [CrossRef]

17. Assem, I.; Simson, D.; Skowronski, A. Elements of the Representation Theory of Associative Algebras; University Press: Cambridge, UK, 2006; Volume 1.

18. Horn, R.A.; Sergeichuk, V.V. Representations of quivers and mixed graphs, Chapter 34. In Handbook of Linear Algebra, 2nd ed.; Hogben, L., Ed.; CRC Press: Boca Raton, FL, USA, 2014.

19. Ringel, C.M. Introduction to the Representation Theory of Quivers. Lectures in the King Abdulaziz University, Saudi Arabia, 2012. Available online: math.uni-bielefeld.de/sek/kau (accessed on 4 February 2021).

20. Sergeichuk, V.V. Representations of simple involutive quivers. In Representations and Quadratic Forms; Akad. Nauk Ukrain. SSR, Inst. Mat.: Kiev, Ukraine, 1979; pp. 127-148. (In Russian)

21. Sergeichuk, V.V. Classification problems for systems of forms and linear mappings. Math. USSR-Izv. 1988, 31, 481-501. [CrossRef]

22. Nazarova, L.A. Representations of quivers of infinite type. Math. USSR-Izv. 1973, 7, 749-792. [CrossRef]

23. Horn, R.A.; Johnson, C.R. Matrix Analysis, 2nd ed.; Cambridge University Press: Cambridge, UK, 2013. 